\title{
Model-stable universality of the air shower electromagnetic component: An approach to solving the mass composition problem
}

\author{
R. Raikin ${ }^{\text {a }}$ T. Serebryakova, A. Lagutin, and N. Volkov \\ Altai State University, 61 Lenin Ave., Barnaul 656049, Russia
}

\begin{abstract}
On the basis of the scaling approach and CORSIKA simulations data the radial scale factor of the lateral distribution of electrons in extensive air showers is confirmed as a potentially effective primary mass estimator, and its sensitivity to hadronic interaction model is investigated. It is shown that improved composition results both on average and event-by-event basis can be achieved taking into account the universality property of air shower development expressed by the relation between the radial scale factor and the longitudinal age parameter. The enhancements of such a theoretically motivated tool for an unbiased cosmic ray composition deduction in a wide primary energy range for current and future (multi-)hybrid air shower measurements are discussed.
\end{abstract}

\section{Introduction}

Uncertainties in hadronic physics and chemical composition are two basic obstacles for understanding the origin of very high energy cosmic rays. A physical interpretation of features of the cosmic ray energy spectrum in terms of sources and propagation properties relies on the assumed mass composition while its robust estimation from extensive air shower (EAS) observables interferes with their sensitivity to nuclei interaction models at energies not accessible with accelerators.

At present, numerous methods and techniques are implemented to infer the mass composition of cosmic rays (e.g. see [1]). They include the analysis of mean values, fluctuations, correlations and even particular features of distributions of different EAS observables such as depth of the shower maximum, muon production depths, total number of electrons and muons at the observation level and local densities far from the shower axis, as well as particle arrival time distributions and the spatial distribution of EAS radio signals. However, despite considerable efforts that have been made in recent years, the composition and its variation with energy remain quite uncertain in almost the entire primary energy range of the cosmic rays available for EAS studies [2-5].

In this paper we examine the efficiency of the formalism describing the lateral distribution function (LDF) of EAS electrons as a scale-invariant and its dependence on the shower longitudinal development stage, that reflects EAS universality properties, for reducing the uncertainties in the current analysis and for improving the estimation of the mean mass composition at a certain energy or the primary particle type in the case of individual showers.

In the following section we give a brief overview of the scaling approach for LDF, proposed in [6-9], and

a e-mail: raikin@theory.asu.ru an outline for the following simulations data analysis. In Sect. 3 we present the results of CORSIKA simulations of EAS carried out with EPOS LHC and SIBYLL nuclei interaction models: distributions of depth of shower maximum $X_{\max }$ and radial scale factor $R_{0}$ for vertical showers initiated by protons and iron nuclei, correlations between $R_{0}$ and the longitudinal age parameter, $s$. Section 4 contains the discussion and final conclusions.

\section{Radial scaling of the electron component and universality in EAS development}

One of the key EAS quantities necessary for basic shower parameters reconstruction is the lateral distribution of charged particles at a fixed observation depth, $X$. The exact form of LDF has been debatable for decades. The majority of analytical parametrizations of LDF of different EAS components is traditionally based on the well known Nishimura-Kamata-Greizen (NKG) function [10] originally obtained for electromagnetic cascade showers:

$$
\begin{aligned}
\rho(r ; E, s)= & \frac{N(E, s)}{r_{0}^{2}} \frac{\Gamma(4.5-s)}{2 \pi \Gamma(s) \Gamma(4.5-2 s)} \\
& \times\left(\frac{r}{r_{0}}\right)^{s-2}\left(1+\frac{r}{r_{0}}\right)^{s-4.5} .
\end{aligned}
$$

Here $\rho(r ; E, s)$ is the local particle density at a radial distance, $r$, from the core position in the shower with primary energy, $E$, and the longitudinal age parameter, $s, N(E, s)$ - total number of particles at the observation depth (shower size), $r_{0}$ - shower scale radius, which does not depend on the primary particle type and energy (originally - the Molière unit $r_{M}$ ). Various modifications of the NKG function, such as introducing different fixed scale factors, lateral $\left(s_{\perp}\right)$ or local $(s(r))$ age parameters and also

(C) The Authors, published by EDP Sciences. This is an Open Access article distributed under the terms of the Creative Commons Attribution License 4.0 (http://creativecommons.org/licenses/by/4.0/). 
generalizations of the function by using a third power-law term were suggested. A comprehensive review is beyond the scope of this paper (some discussions can be found in e.g., [11-13]).

A different theoretically motivated approach, so-called scaling formalism, was proposed in our papers [6-9] for the lateral distribution of electrons in both electromagnetic and hadronic showers:

$$
\rho(r ; E, X)=\frac{N(E, X)}{R_{0}^{2}(E, X)} F\left(\frac{r}{R_{0}(E, X)}\right) .
$$

Here the radial scale factor, $R_{0}$, in contrast to the commonly used $r_{M}$, depends on the primary particle type, the shower age and (in case of extensive air showers) properties of hadronic interactions. The function $F(x)$ is the common scaling part of LDF. According to our calculations based on a semi-analytical approach $[6,7,9]$, the factor $R_{0}$ is equal to the root mean square radius of the electron component, $R_{\mathrm{ms}}$, which is defined in a standard way as

$$
R_{\mathrm{ms}}(E, X)=\left(\frac{2 \pi}{N(E, X)} \int_{0}^{\infty} r^{2} \rho(r ; E, X) r d r\right)^{1 / 2}
$$

and the following expression suggested in [8] for $F(x)$ could be used

$$
F(x)=C x^{-\alpha}(1+x)^{-(\beta-\alpha)}\left(1+(x / 10)^{\gamma}\right)^{-\delta},
$$

where $C=0.28, \alpha=1.2, \beta=4.53, \gamma=2.0, \delta=0.6$.

On the basis of simulations using the simplified hadronic generator, it was also found $[9,14,15]$ that the relation between the lateral shape of the electron distribution and the longitudinal shower age can be expressed by the $R_{0}(s)$ functional dependence, that is a consequence of shower universality properties [16].

\section{Results}

Monte-Carlo simulations of EAS initiated by protons and iron nuclei in the energy range $10^{15} \div 10^{19} \mathrm{eV}$ were performed using CORSIKA v.7.4100 [20] with EPOS LHC v.3400 and SIBYLL v.2.1 (FLUKA 2011.2c.2) hadronic interactions models. In order to get reliable data on electron LDF at very large distances from the shower core the thinning level and particle weight limit were set as $\varepsilon_{t h}=10^{-8}$ and $\omega=10^{2}$ respectively.

The analysis showed that for the averaged lateral distributions the whole set of calculated electron densities are reproduced by the scaling representation (Eq. (2)) within $20 \%$ in relative discrepancy over the interval of scaling variable $x=r / R_{0}=(0.05 \div 25)$, corresponding to the region of radial distances from $r \sim 10 \mathrm{~m}$ to $r \sim(2 \div 4) \mathrm{km}$. For smaller distances discrepancies become large. It does not allow to use the scaling approach for shower size evaluation from the experimentally measured local densities far from the axis. This limitation of the scaling approach was also noted in [17-19]. Nevertheless, scaling formalism provides precise description of the shape of lateral distribution of electrons at radial distances, where local densities can be measured by ground detectors of large air shower arrays. It should
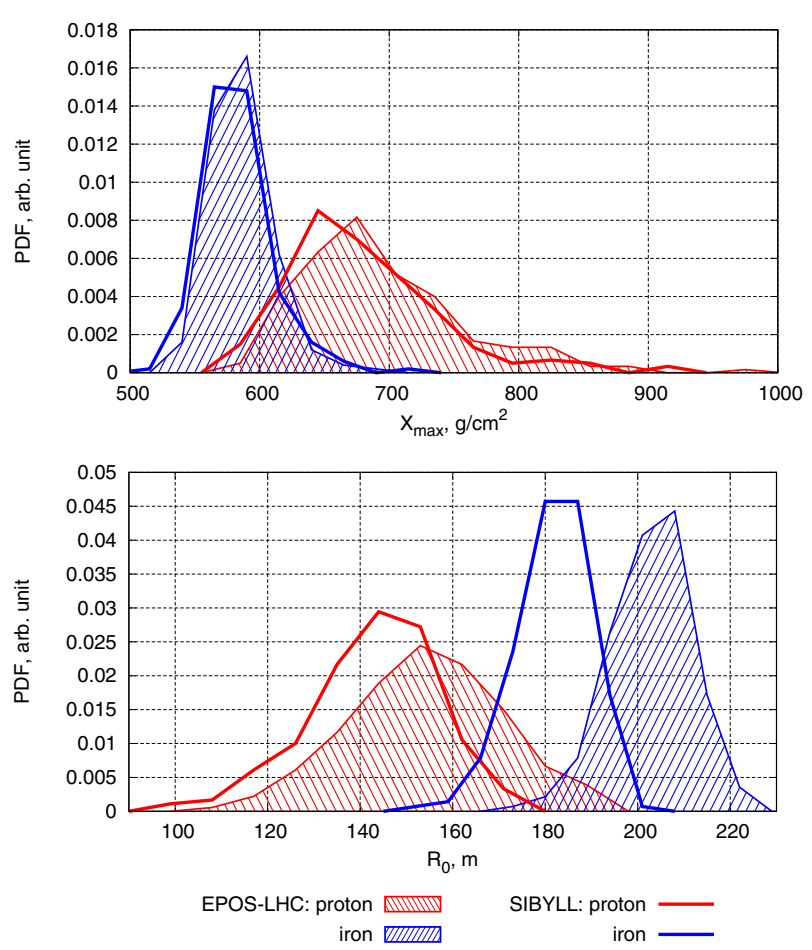

Figure 1. Distributions of depth of shower maximum $X_{\max }$ (top) and radial scale factor $R_{0}$ (bottom) at sea level for $10^{17} \mathrm{eV}$ vertical showers initiated by protons (red) and iron nuclei (blue).

be particularly emphasized that the dependence of LDF on hadronic interaction models is completely governed by the variation of the single parameter, $R_{0}$.

Radial scale factors were evaluated for both average and individual showers as parameters of simulated LDFs fitting. Note, that to obtain the unbiased estimates of $R_{0}$ an iterative procedure was implemented for discrimination of data at distances where the scaling formalism is not valid and also for using fixed distances ranges with respect to the scaling variable $x=r / R_{0}$ for showers of a specific energy and primary particle type. For $F(x)$, the expression of function (4) was used. We have checked out function (4) with a refined set of parameters $\alpha, \beta, \gamma, \delta$ and other representations, including polynomial approximation for $x^{2} F(x)$ giving a considerably better overall fit of simulated data. It was found that the resulting $R_{0}$ values demonstrate only a weak dependence on the explicit form of $F(x)$ choosing for fitting.

The distributions of depth of the shower maximum, $X_{\max }$, and radial scale factor, $R_{0}$, at sea level for $10^{17} \mathrm{eV}$ vertical showers initiated by protons and iron nuclei are shown in Fig. 1. 200 simulated showers are included in each data set. Results for EPOS LHC and SIBYLL interaction models are shown with shaded and open distributions respectively. The figure clearly shows that $R_{0}$ estimated from simulations is more sensitive to both primary mass and interaction model in comparison with $X_{\max }$.

Figure 2 shows the scale factor, $R_{0}$, for average EAS of fixed energy at sea level versus the longitudinal shower age, $s$, defined in terms of a classic cascade theory as $s\left(X, X_{\max }\right)=3 /\left(1+2 X_{\max } / X\right)$. At the first stage electrons produced from the decay of low energy muons were eliminated from local particle densities. In this case electron LDF is a pure superposition of partial 


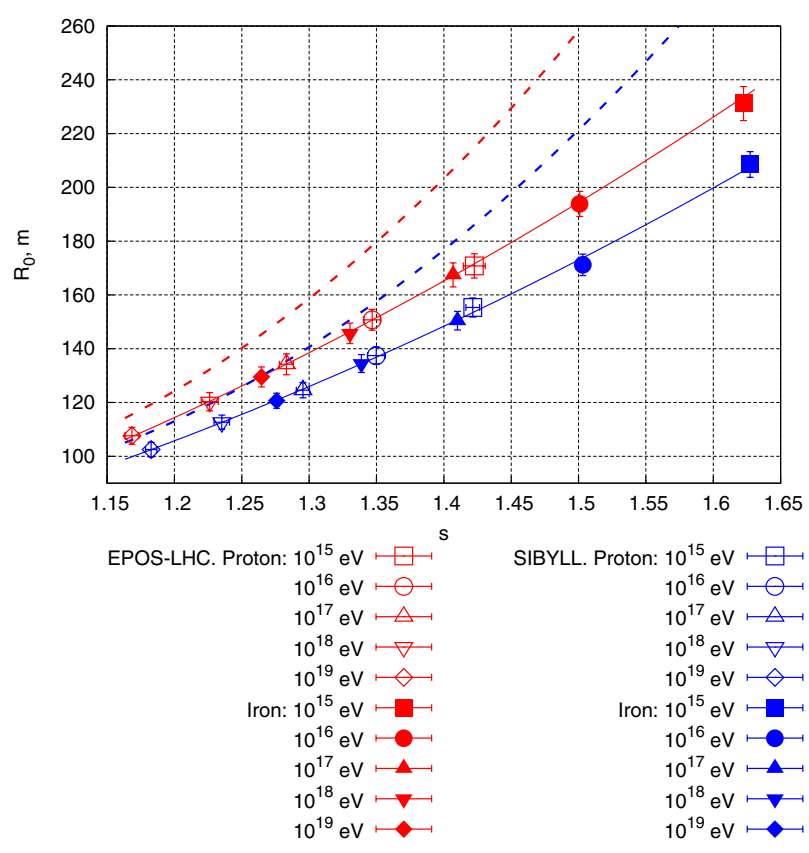

Figure 2. Radial scale factor, $R_{0}$, for average EAS of fixed energy as a function of shower age, $s$, (see text for details).

electromagnetic subshowers definitely possessing scaling and universality features. This data is shown by symbols and solid lines of red (EPOS LHC) and blue (SIBYLL) color. When the contribution of electrons from muon decays is taking into account the LDF become narrower at very large distances, which results in increased $R_{0}$. This effect wanes with energy. The corresponding data is presented by dashed lines in Fig. 2. It is important that in both cases there is a functional dependence $R_{0}(s)$, i.e., one-valued relation between parameters of the shower lateral $\left(R_{0}\right)$ and the longitudinal $(s)$ development stage representing the air shower universality $[14,15]$.

One can see from Fig. 2, that the rate of decrease of $R_{0}$ with energy is almost equal for the two interaction models used in the calculations. Thus the $\partial R_{0} / \partial \log E$ value can be suggested $[9,21-24]$ to be a model-independent measure of primary composition variations with energy similar to the widely used elongation rate approach.

Finally we investigated the possibility of using $R_{0}$ together with $X_{\max }$ for a two-component event-by-event analysis available in the case of hybrid measurements by the surface array and fluorescent telescopes. In Fig. 3 correlations and fluctuations of $R_{0}$ and $s$ at sea level are shown for $10^{17} \mathrm{eV}$ vertical proton- and iron-induced showers simulated with the EPOS LHC model. Strong anticorrelation useful for primary mass discrimination in individual showers is observed.

\section{Discussion}

Discrepancies between mass composition estimates, obtained by different methods from different air shower arrays data, are probably caused by the sum of instrumental and methodical systematic biases, as well as a strong model dependence of observables using as primary mass indicators (mostly in case of muon component characteristics), the inability of effective discrimination of electromagnetic and muon contributions to the ground

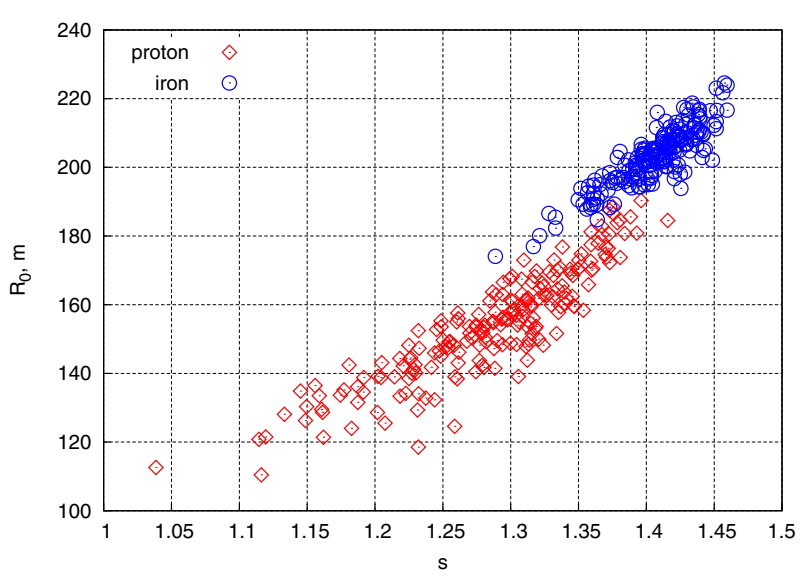

Figure 3. $R_{0}$ vs. $s$ at sea level scatter plot. Data for 200 simulated vertical showers initiated by protons (red diamonds) and iron nuclei (blue circles) of $10^{17} \mathrm{eV}$ (EPOS LHC model) is given.

EAS signal, the lack of understanding of meteorological effects and specific detectors properties etc.

A possible solution to the problem might be achieved with refined multi-hybrid measurements by existing EAS arrays taking into account the capabilities of their anticipated upgrades (e.g., separate detection of electromagnetic and muon contributions to the ground signal by "Auger Prime" [25]) together with generalizations of the analysis by revealing universal features, new parameters and functionals that exhibit weak sensitivity to the interaction model being good primary mass indicators.

The slope of the lateral distribution of EAS charged particles far from the shower core is known to be a primary mass discriminator. But it is rarely used in recent experimental works because precision measurements of the shape of the lateral distribution can not be realised by air shower arrays with large separation between ground stations. On the other hand the important advantage of the LDF as a source of information about mass composition is that ground detectors of charged particles provide an order of magnitude higher duty cycle in comparison with atmospheric telescopes. It is important to note here that using the characteristics of the muon component as basic composition estimates faces the "muon excess problem" [26-28], which requires extended investigations.

\section{Conclusions}

On the basis of the analysis of CORSIKA simulations we show that, when an adequate model-independent description of the lateral distribution of electrons in the framework of scaling formalism is adopted, the single integral parameter of the lateral distribution - scaling factor $R_{0}-$ is a potentially effective primary mass estimator. When combining with the data on longitudinal shower age, it provides improved composition results for both averaged shower measurements and on an event-byevent basis.

The proposed approach exhibits promises as a tool for both primary composition studies and hadronic interaction models tests in a wide primary energy range. It could be implemented for present and future (multi-)hybrid EAS observations, especially in realising the potential of Auger 
and Telescope Array observatories upgrades, as well as for re-analysis and cross-calibration of the data collected from different air shower arrays.

This work was supported by Russian Foundation for Basic Research (grant \#16-02-01103 a).

\section{References}

[1] K.-H. Kampert, M. Unger, Astropart. Phys. 35, 660-678 (2012)

[2] A. Haungs, Physics Procedia 61, 425-434 (2015)

[3] A. Haungs, J. Phys.: Conference Series 632, 012093 (2015)

[4] K.-H. Kampert, P. Tinyakov, Comptes Rendus Physique 15, 318-324 (2014)

[5] S. Buitink et al., Nature 531, 70-73 (2016)

[6] A.A. Lagutin et al., Proc. 25 ICRC, Durban 6, 285-288 (1997)

[7] A.A. Lagutin et al., Nucl. Phys. B (Proc. Suppl.) 60B, 161-164 (1998)

[8] A.A. Lagutin, R.I. Raikin, Nucl. Phys. B (Proc. Suppl.) 97B, 274-277 (2001)

[9] A.A. Lagutin et al., J. Phys. G: Nucl. Part. Phys. 28, 1259-1274 (2002)

[10] K. Greizen, Ann. Rev. Nucl. Sci. 10, 63-108 (1960)

[11] W.D. Apel et al., Astroparticle Physics 24, 467-483 (2006)

[12] R.K. Dey, A. Bhadra, J.N. Capdevielle, J. Phys. G: Nucl. Part. Phys. 39, 085201 (2012)
[13] R. Dey, S. Dam, Eur. Phys. J. Plus 131, 402 (2016)

[14] A.A. Lagutin, R.I. Raikin, T.A. Serebryakova, Bull. Russ. Acad. Sci. Phys. 77(5), 623-625 (2013)

[15] A.A. Lagutin, R.I. Raikin, T.A. Serebryakova, J. Phys.: Conf. Ser. 409, 012092 (2013)

[16] P. Lipari, Phys. Rev. D 79, 063001 (2009)

[17] N.N. Kalmykov, G.V. Kulikov, V.P. Sulakov et al., Bull. Russ. Acad. Sci. Phys. 71, 522-525 (2007)

[18] N.N. Kalmykov, G.V. Kulikov, V.P. Sulakov et al., Moscow Univ. Phys. 62, 31-34 (2007)

[19] Yu.A. Fomin et al., Nucl. Phys. B (Proc. Suppl.) 175-176, 334-337 (2008)

[20] D. Heck et al., Report FZKA 6019 (Forschungszentrum Karlsruhe, 1998)

[21] R.I. Raikin et al., Nucl. Phys. B (Proc. Suppl.) 75-176, 559-562 (2008)

[22] R.I. Raikin et al., Nucl. Phys. B (Proc. Suppl.) 196, 383-386 (2009)

[23] R.I. Raikin, A.A. Lagutin, Bull. Russ. Acad. Sci. Phys. 75(3), 305-308 (2011)

[24] R.I. Raikin, A.A. Lagutin, Proc. of 32 ICRC, Beijing, China 1, 299-302 (2011)

[25] A. Aab et al. (Pierre Auger Collaboration), arXiv: 1604.03637 (2016)

[26] T. Abu-Zayyad et al. (HiRes-MIA Collaboration), Phys. Rev. Lett. 84, 4276 (2000)

[27] A. Aab et al. (Pierre Auger Collaboration), Nucl. Instrum. Methods Phys. Res., Sect. A 798, 172 (2015)

[28] A. Aab et al. (Pierre Auger Collaboration), Phys. Rev. Lett. 117, 192001 (2016) 